\title{
EL PROCESO DE INTEGRACIÓN DE LOS MERCADOS FINANCIEROS EN EUROPA
}

Por: Ignacio López Domínguez*

\section{Resumen}

En este artículo se estudia el proceso y la situación en la que se encuentra la integración de los mercados financieros en el ámbito europeo. Para ello, se repasa brevemente la situación de los mercados financieros españoles para, posteriormente, analizar la conveniencia de unificar los distintos mercados financieros europeos, así como sus repercusiones en una futura bolsa europea.

\section{Palabras clave}

Mercado financiero, "generalización mercados financieros», mercado de valores, mercado de capitales, mercados financieros comunitarios

\section{INTRODUCCIÓN}

Los mercados financieros a escala global atraviesan unos momentos complicados por el contexto económico actual, caracterizado por la inestabilidad. A pesar de ello, en la Unión Europea se está realizando un gran esfuerzo para armonizar la legislación relativa al funcionamiento de los mercados, con el objetivo de lograr una futura integración.

Aunque la mayoría de los estados que componen la Unión comparten una moneda única, los mercados financieros se rigen por normas diversas, distintos sistemas de contratación y estructuras de propiedad.

La armonización legal es necesaria para la integración de los mercados. No obstante, serán las empresas gestoras de estos quienes establezcan sus estrategias, de cara a posibles fusiones en el futuro.

\footnotetext{
* Economista. Doctor en ciencias empresariales. Profesor asociado de la Universidad Complutense de Madrid; profesor de finanzas de la Universidad Antonio de Nebrija; Presidente de la Comisión de Mercados e Instrumentos Financieros del Colegio de Economistas de Madrid; Vicepresidente de la Asociación Internacional de Estudios sobre Management, consultor asociado de CLI Consultores y Consejero Delegado de Neldom Solutions.
} 


\section{LA INTEGRACIÓN DE LOS MERCADOS FINANCIEROS ESPAÑOLES}

\subsection{Características del entorno}

No se puede observar un gran cambio de los mercados financieros españoles hasta 1978, motivado por la crisis industrial que asoló a la economía española en la segunda mitad de la década de los años setenta y en los errores de gestión de una serie de bancos, creados a principios de la década, que no fueron capaces de superar la fase descendente del ciclo económico. Así, esta crisis puso de relieve la necesidad de reformar los mercados financieros para hacerlos más flexibles, transparentes y consecuentes con el rigor en la gestión. También enseñó que era posible hacer frente a las consecuencias sin que se produjeran los cataclismos económicos y financieros que ocurrieron en otros lugares y, ello, gracias al diseño de un Fondo de Garantía de Depósitos y una Ley de disciplina Bancaria que han demostrado, en la práctica, su eficacia.

En cuanto a la valoración de los 25 últimos años del sector financiero español hay que indicar que lo más sobresaliente ha sido, junto con la modernización del sistema financiero, la transformación que se ha producido en los bancos. Otro aspecto por destacar en estos últimos años es la internacionalización de nuestras entidades, la salida del mercado español en busca de nuevos horizontes. De modo, que actualmente, las tendencias más importantes de los mercados financieros son la transparencia y liberalización de las operaciones, la internacionalización de los mercados locales y la implantación de las nuevas tecnologías.

\section{Abstract}

In this article, the process and situation in which the integration of financial markets are involved in Europe are described. For this reason, the situation of the Spanish market is briefly reviewed to analyze the convenience for unifying the different European financial markets as well as the consequences for a future European stock market.

\section{KEY WORDS}

Financial market- generalization of financial markets- value marketscapital market- community financial markets. 
La transparencia y la liberalización de los mercados financieros es una consecuencia directa del pensamiento económico de nuestra época, pues para que un mercado financiero sea eficiente es necesario que se regulen las actividades monopolísticas y las destinadas a alterar el precio de los títulos, pero sin interferir en la libertad casi total de los inversores. De esta forma, la mayor libertad de los inversores e intermediarios, se ha acompañado por una mayor vigilancia por parte de las autoridades para evitar fraudes y manipulaciones, penalizando la negociación con información privilegiada o confidencial ("insider trading"), etc. Así, en España, el órgano de supervisión y control es la Comisión Nacional del Mercado de Valores (CNMV), concretamente, es el organismo creado por la Ley del Mercado de Valores de 1988 para supervisar todos los mercados financieros nacionales.

La internacionalización es otro signo de nuestra época, tanto en el ámbito económico como social, con una tendencia clara hacia una globalización de las actividades económicas o sociales. La apertura financiera de todos los países hacia el exterior, aboliendo las restricciones a la libre movilidad de capitales, ha sido una necesidad para financiar la mayoría de economías nacionales. El aumento de las inversiones de los no residentes o inversores extranjeros ha sido constante en todos los mercados, tanto en los países más desarrollados, como en los que están abriendo ahora sus primeros mercados financieros. Así, por ejemplo, a los mercados más desarrollados acuden inversores del tercer mundo en busca de seguridad y, por el contrario, a los mercados emergentes, acuden inversores de los países más desarrollados, en busca de una mayor rentabilidad, a pesar del mayor riesgo que presentan.
Esta internacionalización de los mercados, introduce el factor de la competencia en los mismos e impone su modernización y especialización en los segmentos donde son más competitivos. Pero esta globalización de los mercados también aumenta su vulnerabilidad ante crisis lejanas, que a pesar de tener unas consecuencias mínimas en el campo puramente económico nacional, encuentra unos inversores muy sensibilizados ante los problemas de otros mercados financieros, con lo que crisis económicas locales se convierten en crisis financieras mundiales.

Por último, la implantación de nuevas tecnologías ha cambiado totalmente los mercados financieros, produciendo dos cambios sustanciales en los mismos: la desaparición física de los títulos, sustituidos por anotaciones en cuentas informáticas y la multidifusión de las cotizaciones en tiempo real a través de complejas redes informáticas. La desaparición del título como documento físico, ha agilizado la liquidación de las operaciones, aumentando la seguridad de las mismas. Por su parte, las posibilidades de difusión de la información en tiempo real mediante pantallas de ordenador, han aumentando la transparencia y popularidad de los mercados financieros y ha atraído, hacia los mismos, nuevos inversores que confían en su seguridad y rentabilidad actuales.

Además, las nuevas tecnologías han permitido la deslocalización física de las Bolsas de Valores, de forma que ahora están donde existe la pantalla de un terminal de ordenador, por lo que el acceso de los inversores a los mercados, en tiempo real, es casi total. Sin embargo, la implantación de las nuevas tecnologías no se detiene; ahora, la extensión de Internet abre nuevas posibilidades de aproximación y transparencia, insospechadas hace pocos años. 


\subsection{Proceso de integración: antecedentes}

Tras la liberalización de los mercados financieros españoles, había que hacer algo antes de que se produjese la inevitable consolidación operativa de los distintos mercados europeos. Por ello, el año 2000 empezaba un proceso de integración de las sociedades Meff, Aiaf y Senaf. Se trata de una operación de fusión de tres sociedades rectoras, creando un holding común, para ofrecer un servicio integrado, en las mejores condiciones de calidad y coste.

Figura 1. Organigrama de Bolsas y Mercados Españoles

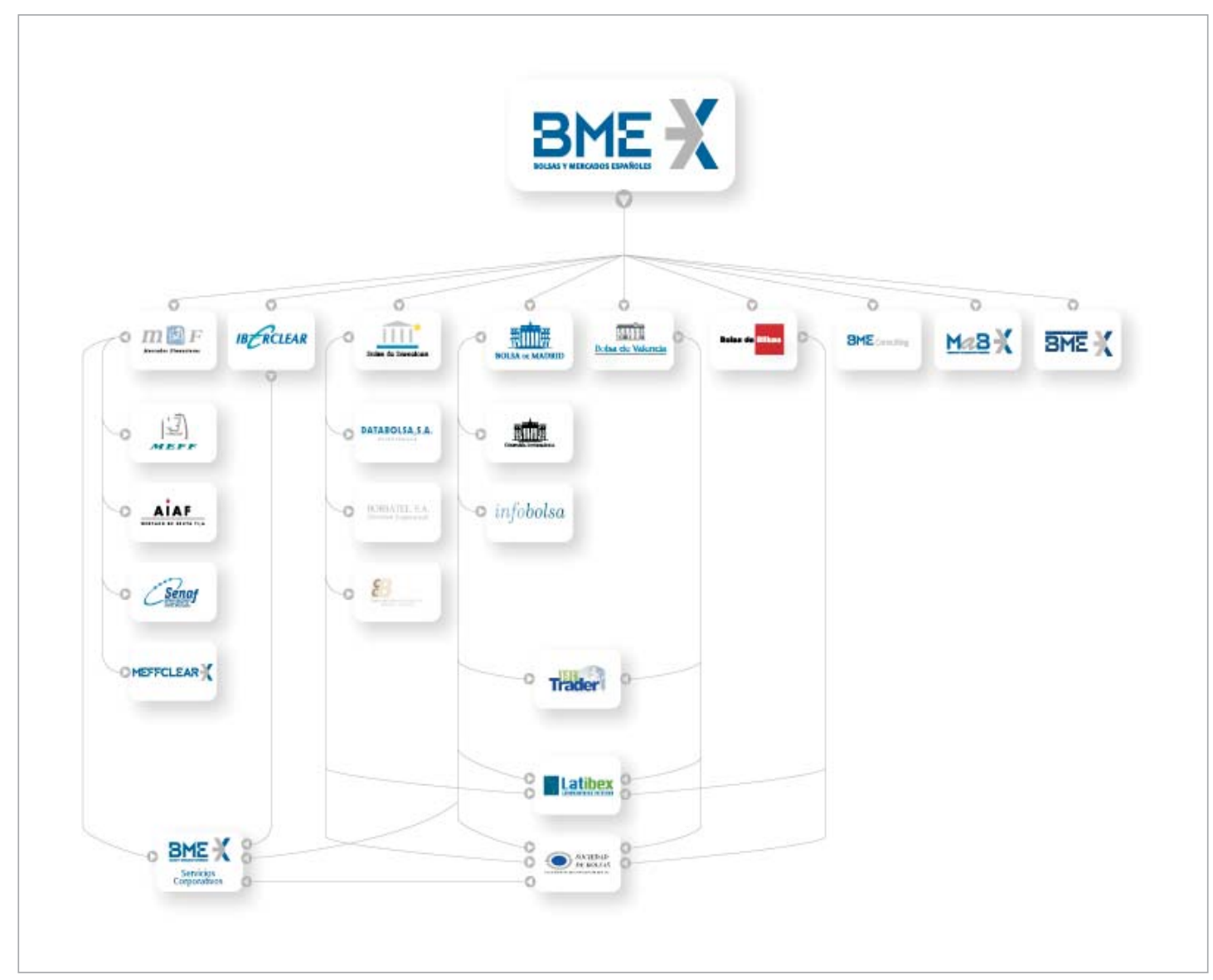

Fuente: Bolsas y Mercados Españoles (BME)

Grupo mF es el Holding resultante de la integración del Mercado español de Futuros y Opciones (MEFF), el Mercado español de Deuda Corporativa (AIAF) y la plataforma electrónica de negociación de Deuda Pública (SENAF).
Dicha integración se produjo en el año 2001 como respuesta de los tres mercados a las exigencias de integración que impone la globalización de los mercados, lográndose así conjuntar los recursos humanos y tecnológicos de dichas sociedades, reducir los costes para 
los Miembros de los mercados, mejorar la calidad del servicio y ofrecer soluciones técnicas e informáticas integradas.

Bolsas y Mercados Españoles (BME) integra las diferentes empresas que dirigen y gestionan los mercados de valores y sistemas financieros en España. Agrupa, bajo la misma unidad de acción, decisión y coordinación, los mercados de renta variable, renta fija, derivados y sistemas de compensación y liquidación españoles. El grupo BME está integrado por la Bolsa de Barcelona, Bolsa de Bilbao, Bolsa de Madrid, Bolsa de Valencia, MFMercados Financieros, en cuyo seno se encuentra AIAF e lberclear.

BME es la respuesta de los mercados españoles al nuevo entorno financiero internacional donde los inversores, intermediarios y empresas demandan una creciente gama de servicios y productos en un marco de seguridad, transparencia, flexibilidad y competitividad.

La unión de esfuerzos permite a los mercados españoles alcanzar una dimensión de primer nivel en el ámbito europeo, una estructura diversificada que engloba toda la cadena de actividad en los mercados de valores, desde la contratación hasta la liquidación, pasando por la difusión de información y servicios informáticos. Este tamaño y estructura permite un mejor aprovechamiento de recursos, la reducción de los costes y la racionalización de los servicios ofrecidos. De esta manera, BME desempeñará un papel decisivo en la configuración del mapa bursátil europeo.

La creación de Bolsas y Mercados Españoles supone en la práctica la integración de todas esas entidades en una sola que será responsable de su unidad de acción, decisión y coordinación estratégicas. Según el objeto social de la nueva sociedad, para lograrlo, habría que desarrollar las mejoras operativas, funcionales y estructurales que fueran consistentes con la consecución de unos mayores niveles de eficiencia global de los mismos y con su potenciación de cara al exterior, sin perjuicio de que las entidades individuales integradas bajo la sociedad "holding" mantuviesen su identidad, su capacidad operativa, sus órganos de administración y sus equipos gerenciales y humanos.

\section{LA CONSTRUCCIÓN DE UN MERCADO ÚNICO DE LOS SERVICIOS FINANCIEROS EN LA UE.}

E ste largo camino se inicia con el propio nacimiento de la Comunidad, encontrando un impulso definitivo con la introducción del euro. En junio de 1998, en el Consejo Europeo de Cardiff, los jefes de gobierno de la Unión Europea encargaron a la Comisión la elaboración de un Plan de Acción para conseguir la unificación de los mercados financieros comunitarios (PASF Plan de Acción de los Servicios Financieros). Dicho Plan consta de 43 medidas -legislativas y no legislativas- y tiene como objetivo la consecución de un mercado único de los servicios financieros en la UE.

\subsection{La libre circulación de capitales}

Cuando el 25 de marzo de 1957 nace en Roma la Comunidad Económica Europea (CEE), se da un paso de gigante en el proceso de 
construcción de una Europa Unida. De la simple cooperación que suponía la Organización Europea de Cooperación Económica (1948), se había pasado en menos de tres años a la integración del carbón y del acero y en otros seis a una integración total de las economías.

Como exigían las prescripciones de la ciencia económica, el objetivo prioritario de la CEE fue el establecimiento de un mercado unificado entre los países miembros, en el que la libre circulación de mercancías fuese una realidad y donde frente al exterior existiese una única tarifa aduanera. El principio de la libre circulación se extendió a los capitales, la mano de obra y los servicios, porque los argumentos que la avalan, son también válidos para los factores de producción. Para consolidar un mercado común de estas características, era necesario eliminar los obstáculos arancelarios y no arancelarios a los intercambios comerciales, la puesta en marcha de políticas comunes entre los Estados miembros, así como la aplicación rigurosa del principio de competencia.

La libre circulación de capitales y de pagos exige la supresión de las restricciones a los movimientos de capital, así como la liberalización de los medios de pago. Para comprender cabalmente lo que esto significa, conviene recordar que son movimientos de capital las transacciones financieras que no constituyen retribuciones o remuneraciones por entregas de bienes o prestaciones de servicios e incluyen, en consecuencia, las inversiones (directas e inmobiliarias) así como las operaciones personales. En otras palabras, los movimientos de capital son transferencias de valores de un estado a otro. Por su parte, la liberalización de pagos se refiere a las retribuciones por prestaciones en el marco de una transacción o de una transferencia de divisas ya se hagan en efectivo, en cheques o en divisas.
El proceso de construcción europea sufre una importante ralentización, hasta que en 1984 se publica el Libro Blanco sobre la terminación del mercado interior, cuyo propósito era acabar con los tres tipos de barreras que venían frenando el desarrollo del mercado común: físicas, técnicas y fiscales.

El Libro Blanco fue la base sobre la que el Consejo Europeo celebrado en Luxemburgo el 2 y 3 de diciembre de 1985 aprobó la llamada Acta Única Europea, que se firmaría el 28 de febrero de 1986 y entraría en vigor el 1 de julio de 1987.

Las ideas centrales del Acta Única eran tres: culminar el mercado interior convirtiendo el territorio comunitario en un espacio sin fronteras donde la libre circulación de mercancías, personas, servicios y capitales estuviese garantizada; consagrar la cohesión económica y social como uno de los pilares básicos de la construcción europea y poner los raíles para la unión económica y monetaria.

En el año 1993 se adoptó una directiva clave para la construcción del mercado interior de servicios financieros: la Directiva 93/22/ de 10 de mayo de 1993 de Servicios de Inversión. La citada Directiva incluye dos novedades importantes:

- Introduce el pasaporte comunitario para las empresas de servicios de inversión (sociedades y agencias de valores, bancos y sociedades gestoras de cartera), lo que les permite prestar servicios financieros en toda la Unión con la autorización del supervisor de su país de origen.

- Recoge, dentro del ámbito de la supervisión, el principio de país de origen para la supervisión prudencial de las empresas de servicios de inversión y el de país de destino (o país de acogida) para la prestación del 
servicio financiero. Por aplicación del primero de estos principios, la solvencia será vigilada por el supervisor del país de origen de la entidad financiera. Por aplicación del segundo, las normas de conducta aplicables a las relaciones entre la entidad financiera y los inversores serán establecidas por el país de residencia de estos últimos.

El último de los pasos para la construcción de un mercado único de los servicios financieros, antes del Plan de Acción de Servicios Financieros, lo da el Plan de Acción para el Mercado Único (Consejo de Florencia, junio de 1996), que establece una lista de medidas para mejorar su funcionamiento.

Entre estas medidas interesa destacar las siguientes:

- Acelerar la transposición de las Directivas relativas al Mercado Único, siendo los sectores más retrasados la contratación pública y los seguros.

- Avanzar en el proceso de armonización fiscal, dada la relación existente entre la libre circulación de capitales y la imposición sobre el ahorro.

\subsection{La Unión Económica y Monetaria}

Además de sus logros en materia de mercado interior, el Acta Única constituyó la rampa de lanzamiento de la Unión Económica y Monetaria, pues al igual que no puede existir un verdadero mercado único sin una moneda única, ésta no puede desarrollarse sin un Mercado Único que le sirva de base.

En abril de 1989, el informe del Comité Delors preveía la realización de la UEM en tres fases: el objetivo de la primera, entre junio de 1990 y enero de 1992, consistía en reforzar la cooperación entre los bancos centrales; la segunda, conllevaba la creación de un Sistema Europeo de Bancos Centrales (SEBC), así como la transferencia progresiva del poder de decisión en materia de política monetaria a las instituciones supranacionales; por último, durante la tercera fase, se fijarían de forma irrevocable las paridades de las monedas nacionales y éstas se sustituirían por la moneda única europea.

En definitiva, la liberalización de los movimientos de capitales, junto con la introducción del euro, pusieron de manifiesto la necesidad de avanzar en la integración de los mercados financieros. En efecto, la Unión Económica y Monetaria no puede ofrecer a la sociedad europea los beneficios esperados en términos de crecimiento, empleo y competitividad, si no viene acompañada de la integración de los mercados financieros. Esta necesidad de integración parece, además, necesaria si la Unión Europea quiere subsistir con éxito en una economía globalizada.

\subsection{La globalización}

La globalización se identifica con la interdependencia creciente de las economías nacionales, provocada por el incremento del volumen y de la variedad de las transacciones transfronterizas de bienes o servicios, así como por el vertiginoso aumento de los flujos internacionales de capitales y por la difusión acelerada y generalizada de la tecnología.

La libre circulación de capitales, la introducción del euro y la globalización de los mercados provocaron una profunda transformación del sector financiero europeo (bancos, instituciones financieras, empresas de servicios de inversión y agencias de seguros) que se manifestaba en los siguientes aspectos:

- Un mayor desarrollo de los mercados de capitales, sobre todo de las inversiones del 
sector privado, ya sean acciones o renta fija privada y una paralela reducción de la deuda pública (consecuencia del Pacto de Estabilidad que impone límites severos al endeudamiento de las Administraciones Públicas)

- Una supremacía absoluta de los vehículos institucionales de ahorro (organismos de inversión colectiva y fondos de pensiones) frente a la alternativa de inversión individual de cada ahorrador.

- Una ampliación de la actividad tradicional de la banca (prestamista de empresas o consumidores) para extenderse a la gestión de patrimonios de terceros (incluidos los fondos de inversión) en valores mobiliarios.

- Un cierto grado de especialización de los sistemas financieros nacionales (el bono alemán constituye la referencia para la deuda soberana, al mismo tiempo que los bonos "Pfandbrief" empiezan a serlo en la deuda privada); así como la aparición de mercados alternativos de papel a corto? con mayor rentabilidad, pero también con menor seguridad, que podrían configurar un binomio de rentabilidad-riesgo distinto al que había venido siendo habitual.

\subsection{El plan de acción de los servicios financieros}

La Comisión publicó en 1998 una Comunicación - "Servicios Financieros: Establecimiento de un marco de adaptación

" - en la que se indicaban diversos aspectos que requerían medidas urgentes para garantizar el pleno aprovechamiento de las ventajas derivadas de la moneda única y para conseguir un funcionamiento óptimo del mercado financiero europeo. En dicho documento se puso de relieve la necesidad de actuar en cinco campos diferentes:
- Dotar a la UE de un dispositivo legislativo más claro y moderno, capaz de responder a los nuevos desafíos.

- Acabar con la fragmentación del mercado de capitales para reducir el coste de constitución de capital en los mercados de la UE.

- Permitir a los prestadores de servicios y usuarios aprovechar las oportunidades ofrecidas por un mercado financiero único, garantizando a estos últimos un elevado nivel de protección.

- Fomentar una coordinación más estrecha de las autoridades de supervisión y control.

- Desarrollar una infraestructura de la UE integrada que sirva de base para las transacciones financieras al por menor $y$ al por mayor.

El Parlamento Europeo aprobó una resolución el 4 de mayo de 1999 (Informe Fayot) acogiendo favorablemente las propuestas de la Comisión, al mismo tiempo que solicitaba la adopción de medidas específicas en los siguientes ámbitos:

- Presentar una propuesta sobre un procedimiento formalizado de cooperación de los mercados bursátiles nacionales y la creación de un listado panaeuropeo de acciones en lugar de los requisitos nacionales de venta, con la consiguiente actualización de la directiva de 1989 sobre listados y prospectos.

- Elaborar una comunicación sobre un sistema de certificación europea de las mejores prácticas en el sector de la gestión de fondos de inversión.

- Presentar al Parlamento un modelo de agilización de los procedimientos legislativos, en el que se establezcan los principios, el alcance y otros requisitos básicos para mantener actualizada la legislación. 
Estos trabajos sirvieron a la Comisión para preparar el Plan de Acción de Servicios Financieros, diseñado para definir los objetivos y las prioridades, fijar un calendario indicativo de actuación e identificar las acciones necesarias para crear un auténtico Mercado Interior. El Plan de Acción incluye una mezcla de nueva legislación, leyes modificadas, comunicaciones de la Comisión y recomendaciones jerarquizadas en el tiempo, que deberían completarse en el año 2005. El Plan, asimismo, identifica aquellas áreas en las que el bloqueo político ha impedido avances sustanciales, tales como las normas sobre liquidación, ofertas públicas de adquisición, estatuto de la sociedad europea, organismos de inversión colectiva y la venta a distancia de servicios financieros.

Los objetivos estratégicos del Plan de Acción son tres: la consecución de un mercado mayorista único en la UE; el establecimiento de mercados minoristas abiertos y seguros y la revisión de las normas cautelares y de supervisión. Además de estos objetivos, el Plan de Acción establece un objetivo general: el establecimiento de unas condiciones más amplias para un mercado financiero único óptimo.
El Plan de Acción, una vez definidos sus objetivos estratégicos, se preocupa por establecer un calendario óptimo. Según el mismo, las acciones se clasifican, en razón de su urgencia, en los tres siguientes niveles de prioridad:

- Medidas de prioridad 1: son las que se consideran esenciales para aprovechar plenamente las ventajas derivadas de la introducción del euro y para garantizar la competitividad del sector de servicios financieros y de la industria de la Unión, salvaguardando al mismo tiempo los intereses de los consumidores.

- Medidas de prioridad 2, en las que se incluyen aquellas que la Comisión considera importantes para el funcionamiento del mercado único de servicios financieros.

- Medidas de prioridad 3: se trata de medidas en importantes ámbitos en los que existe un consenso general sobre la necesidad de emprender nuevos trabajos, encaminados a configurar una política coherente a finales del período de transición al euro.

\section{LAS VENTAJAS E INCONVENIENTES DE LA NTEGRACIÓN}

$\mathrm{E}_{1}$ euro es, en esencia, una unificación monetaria, uno de cuyos objetivos es garantizar una libre y leal competencia en toda Europa. La plena armonización de los usos del mercado es un ejemplo más de cómo la unificación facilita la comparación entre varios instrumentos financieros, conduciendo también hacia un mercado financiero más eficiente.

Los estudios hasta ahora elaborados coinciden en que, para Europa, unos mercados integrados implicarían mayor eficiencia en las transacciones financieras, al amparo de economías de escala y de red. De ahí se derivaría probablemente un menor coste de capital para los emisores y prestatarios, así como una mayor tasa de rentabilidad para los ahorradores.

La integración efectiva facilitaría una mayor variedad de transacciones y unos mercados más amplios, profundos y líquidos. Previsiblemente, ello atraería más inversión internacional, diversificaría los plazos de colocación del ahorro, reduciría riesgos y estimularía la innovación. 
Con el respaldo de mercados financieros eficientes, la inversión productiva resultaría impulsada y, a través de ella, se estimularían obviamente el crecimiento económico y la generación de empleo.

La llegada del euro y la internacionalización de los intermediarios financieros y las empresas emisoras son otros factores que juegan a favor de la creación de un mercado único europeo, aunque los expertos recuerdan que el proceso de integración aún deberá salvar algunos obstáculos:

- La distinta fiscalidad de los instrumentos financieros.

- Los diversos procedimientos de compensación y liquidación de valores.

- Las diferencias en el idioma y la cultura financiera de los países que integran la UE.

- La existencia de un cierto proteccionismo de algunas autoridades nacionales sobre determinadas parcelas de su industria financiera.
En resumen, el proceso de integración se enfrenta a diversos problemas, agrupables en tres grandes grupos de obstáculos:

1. Legales. Todavía no se cuenta con una normativa común europea sobre aspectos financieros relevantes, como por ejemplo ocurre en el caso de las fusiones y adquisiciones bancarias.

2. Derivados de una insuficiente iniciativa y coordinación del sector privado.

3. Debidos a inercias nacionalistas de índole político y cultural.

Sobreviven en la UE mercados segmentados de servicios financieros y se producen episodios tan incoherentes como la obstaculización pura y simple de fusiones bancarias pan-europeas o las resistencias a una centralización de los mercados de valores.

En una perspectiva a largo plazo los dos asuntos por tratar son, por un lado, la creación de un solo regulador de los mercados de valores europeos -como la SEC de EEUU-y, por otro, la adopción de estándares básicos de regulación de los mercados de valores europeos.

\section{Conclusiones}

Estamos viviendo actualmente el proceso de $_{\text {de }}$ la integración de los mercados financieros.

La construcción del mercado único se entiende como una eficaz armonización de los mercados, que compiten entre sí y que se articulan en una serie de elementos, como la existencia de adecuados sistemas de interconexión, operativas análogas, normativa homogénea y eficiencia de las transacciones transfronterizas, de modo que los emisores o intermediarios $y$, al fin, los propios inversores, puedan actuar en todo el territorio de la unión sin barreras, como una sola jurisdicción o mercado.

Ahora bien, para que todo lo anterior no se quede en poco más que una mera declaración de buenas intenciones, deberán establecerse los sistemas técnicos de interconexión, de los que en España y en el ámbito de los mercados nacionales tenemos una amplia experiencia; estos deben hacer posible el acceso directo y eficiente de los inversores a cualquiera de los mercados radicados en los países de la Unión. 
La armonización normativa y esa adecuada interconexión darán lugar a la concentración de esos grandes polos de liquidez en competencia entre sí.

No obstante, se plantean diversos problemas de distintas índoles que obstaculizan y retrasan gravemente el proceso, siendo los más importantes las políticas proteccionistas que tienen numerosos países con determinados sectores, las divergencias en materia tributaria y legal $y$, sobre todo, la falta de iniciativa, o la escasa cohesión en las decisiones de los diferentes países.
Es imprescindible la creación de un organismo supranacional que controle y regule este nuevo mercado; sin embargo, los estados e instituciones existentes son reacios a abandonar su posición de poder y control sobre los mercados financieros, por diversos motivos (proteccionismo de los mercados nacionales). La integración es un proceso necesario puesto que estamos inmersos en la era de la globalización, donde hay que ser grande para competir con los grandes.

\section{Bibliografía}

LÓPEZ DOMÍNGUEZ, I (1997): Manual Práctico de los mercados Financieros", ISTPB.

MARTÍNEZ ESTEVÉZ, A. (2000): Mercados Financieros Internacionales, Civitas Ediciones y CAM.

MONTES, P. (1993): La Integración en Europa, del Plan de Estabilización a Maastricht, Editorial Trotta.

OLCESE, A.; MARTÍN, M. (directores) (2004): Estudio sobre la reforma de los mercados financieros europeos. Fundación de Estudos Financieros, Madrid.
ONTIVEROS, E.; BERGES, A.; MANZANO,D.; VALERO, F.J. (1991): Mercados Financieros Internacionales. Biblioteca de Economía Espasa Calpe.

PAREJO GÁMIR, J.A.; CALVO BERNARDINO, A.; PAÚL GUTIÉRREZ, J. (1993): EI Sistema Financiero en los Países de la CE. Editorial AC, Madrid.

PEINADO GRACIA, M ${ }^{\mathrm{a}}$.L. (Coordinadora) (1999): Los mercados financieros españoles y la UEM. Biblioteca Civitas Economía y Empresa, Madrid.

Las Perspectivas de la Economía Mundial, Fondo Monetario Internacional, Washington, mayo 1977. 
